\title{
Leakage-free rapid quenching technique for yeast metabolomics
}

\author{
André B. Canelas · Cor Ras · Angela ten Pierick · Jan C. van Dam · \\ Joseph J. Heijnen · Walter M. van Gulik
}

Received: 25 February 2008/ Accepted: 7 June 2008/Published online: 26 June 2008

(C) The Author(s) 2008

\begin{abstract}
Accurate determination of intracellular metabolite levels requires reliable, reproducible techniques for sampling and sample treatment. Quenching in $60 \%(\mathrm{v} / \mathrm{v})$ methanol at $-40^{\circ} \mathrm{C}$ is currently the standard method for sub-second arrest of metabolic activity in microbial metabolomics but there have been contradictory reports in the literature on whether leakage of metabolites from the cells occurs. We have re-evaluated this method in S. cerevisiae using a comprehensive, strictly quantitative approach. By determining the levels of a large range of metabolites in different sample fractions and establishing mass balances we could trace their fate during the quenching procedure and confirm that leakage of metabolites from yeast cells does occur during conventional cold methanol quenching, to such an extent that the levels of most metabolites have been previously underestimated by at least twofold. In addition, we found that the extent of leakage depends on the time of exposure, the temperature and the properties of the methanol solutions. Using the mass balance approach we could study the effect of different quenching conditions and demonstrate that leakage can be entirely prevented by quenching in pure methanol at $\leq-40^{\circ} \mathrm{C}$, which we propose as a new improved method. Making use of improved data on intracellular metabolite levels we also re-evaluated the need of sub-second quenching of metabolic activity and of removing the extracellular medium. Our findings have serious implications for quantitative metabolomics-based fields such as non-stationary ${ }^{13} \mathrm{C}$ flux analysis, in vivo kinetic modeling and thermodynamic network analysis.
\end{abstract}

A. B. Canelas $(\square) \cdot$ C. Ras · A. ten Pierick .

J. C. van Dam - J. J. Heijnen · W. M. van Gulik

Department of Biotechnology, Delft University of Technology,

Julianalaan 67 2628BC, The Netherlands

e-mail: a.canelas@tudelft.nl
Keywords Quantitative metabolomics . Intracellular metabolite analysis - Rapid sampling . Cold methanol quenching $\cdot$ Leakage $\cdot$ S. cerevisiae

Abbreviations
gDw
$\begin{array}{ll}\text { G6P } & \text { Glucose-6-phosphate } \\ \text { T6P } & \text { Trehalose-6-phosphate } \\ \text { FBP } & \text { Fructose-1,6-bis-phosphate } \\ \text { 6PG } & \text { 6-Phospho gluconate } \\ \text { F6P } & \text { Fructose-6-phosphate } \\ \text { G1P } & \text { Glucose-1-phosphate } \\ \text { M6P } & \text { Mannose-6-phosphate } \\ \text { 2PG } & \text { 2-Phospho glycerate } \\ \text { 3PG } & \text { 3-Phospho glycerate } \\ \text { PEP } & \text { Phosphoenolpyruvate }\end{array}$

\section{Introduction}

The accurate measurement of physiological levels of intracellular metabolites is of prime interest in the study of metabolic reaction networks and their regulation in vivo. Owing mainly to developments in analytical tools, in particular in MS-based techniques, the field of metabolite analysis is undergoing fast expansion. Metabolomics, the systematic analysis of large numbers of low molecular weight compounds from a biological system, is in the process of establishing itself as a global analysis method complementary to transcriptomics and proteomics and is expected to provide major contributions to areas such as functional genomics, toxicology and nutrigenomics. However, whether for quantitative or qualitative purposes, the quality and reliability of metabolomics data will invariably 
depend on the sampling and sample treatment techniques employed, which usually receive relatively little attention. Strikingly, there is no consensus in the literature on the effectiveness or even adequacy of the available techniques for sampling, quenching and extraction of intracellular metabolites from microbial cultures (Dekoning and Vandam 1992; Gonzalez et al. 1997; Hajjaj et al. 1998; Hans et al. 2001; Maharjan and Ferenci 2003; Castrillo et al. 2003; Wittmann et al. 2004; Villas-Boas et al. 2005; Oldiges and Takors 2005; Schaub et al. 2006; Loret et al. 2007; Bolten et al. 2007; Villas-Boas and Bruheim 2007; Mashego et al. 2007).

Many intermediates in metabolic reaction networks have turnover times in the order of seconds, due to the relatively high conversion rates and low metabolite concentrations found in vivo. The need to rapidly quench metabolic activity upon sampling to avoid unwanted changes in intracellular metabolite levels was already recognized and documented by biochemists many decades ago (Wollenberger et al. 1960; Williams et al. 1967; Veech et al. 1969; Faupel et al. 1972). In the case of cell suspension cultures rapid sampling was initially accomplished by sampling broth directly into a cold perchloric acid (PCA) extraction solution followed by a series of freezing-thawing cycles, thus achieving quenching, release of intracellular metabolites and inactivation of enzymes (Harrison and Maitra 1969; Cole et al. 1967; Weibel et al. 1974). However, direct extraction has two major disadvantages: the low concentrations of metabolites in the samples, owing to low biomass densities; and the risk of overestimating intracellular pools, because the metabolites present in the extracellular medium are not removed. The first significant attempt to combine quenching with cell separation involved fast filtration and washing of the cells with $50 \%$ (v/v) methanol at $-40^{\circ} \mathrm{C}$, followed by the extraction step (Saez and Lagunas 1976). Maintaining a very low temperature minimized metabolic activity during filtration while allowing removal of the extracellular medium. This technique was later improved by De Koning and Van Dam, who proposed sampling the broth directly into $60 \%$ (v/v) methanol at $-40^{\circ} \mathrm{C}$ and separating the cells by centrifugation (Dekoning and Vandam 1992). That was a decisive improvement because it allowed sub-second arrest enzymatic activity. This technique has remained mostly unchanged and is still the most widespread method for rapid sampling of microbial cultures (Oldiges and Takors 2005; Mashego et al. 2007).

Perhaps the most critical assumption in the cold methanol quenching method is that intracellular metabolites will remain inside the cells during quenching and centrifugation. If metabolites were to leak from the cells into the methanol solutions, which are discarded, the intracellular levels might be severely underestimated. The original work of De Koning and Van Dam and some of the subsequent literature concluded that metabolite leakage did not occur in yeast (Dekoning and Vandam 1992; Gonzalez et al. 1997), fungi (Ruijter and Visser 1996; Hajjaj et al. 1998) or bacteria (Moritz et al. 2000). However, this assumption is increasingly being questioned after some recent studies, making use of more sensitive MS-based analytical methods, reported the occurrence of extensive losses of intracellular metabolites during cold methanol quenching in yeast (Villas-Boas et al. 2005) as well as bacteria (Wittmann et al. 2004; Bolten et al. 2007). Unfortunately, this issue has not been thoroughly and systematically addressed yet. In addition, if leakage does occur, there is no reliable, validated alternative method, despite some recent efforts in finding substitute quenching solutions (Villas-Boas and Bruheim 2007).

The purpose of this work was to determine quantitatively whether leakage of intracellular metabolites occurs in $S$. cerevisiae during cold methanol quenching and, if so, find a way to prevent it. In contrast with previous method evaluation attempts, we proposed to use only strictly quantitative data from samples obtained in standardized, reproducible culture conditions through well-defined rapid sampling and sample treatment procedures. In addition, a wide range of metabolites was analyzed, to cover different classes of compounds (phosphorylated intermediates, organic acids, aminoacids) and different molecular properties (e.g. molecular weight, polarity). Furthermore, after initial indications of leakage we adopted an even broader approach by measuring metabolite levels in all possible sample fractions and establishing mass balances to be able to trace the fate of the metabolites during cold methanol treatment. Only such a comprehensive approach made it possible to positively conclude on the occurrence of leakage under different conditions and find a way to prevent it. We hope the outcomes of this work will serve those in the metabolomics community by providing an improved quenching method for intracellular metabolite analysis in S. cerevisiae and a standard for method validation in other microorganisms.

\section{Experimental procedures}

\subsection{Solvents and chemicals}

HPLC-grade methanol, ethanol and chloroform were supplied by Baker (The Netherlands). Analytical grade standards were supplied by Sigma.

\subsection{Strain and cultivation conditions}

The Saccharomyces cerevisiae strain used in this study was CEN.PK 113-7D (MATa) (van Dijken et al. 2000). The cells were grown in aerobic carbon-limited chemostat cultures in a 71 fermentor (Applikon, The Netherlands) 
with a working volume of 41 . Unless stated otherwise, defined mineral medium (Verduyn et al. 1992) with $7.5 \mathrm{~g} / \mathrm{l}$ glucose was used, which supported a steady-state biomass concentration of $3.7 \mathrm{~g}_{\mathrm{DW}} / \mathrm{l}$, the dilution rate was $0.1 \mathrm{~h}^{-1}$ and the aeration rate was $0.5 \mathrm{vvm}(120 \mathrm{l} / \mathrm{h})$. Dissolved oxygen tension (DOT) was measured in situ with an oxygen probe (Mettler-Toledo, Switzerland) and $\mathrm{O}_{2}$ and $\mathrm{CO}_{2}$ concentrations in the off-gas were measured at-line using a combined paramagnetic/infrared analyzer (NGA 2000, Rosemount, USA). The $\mathrm{pH}$ was controlled at 5.0 with $4 \mathrm{M}$ $\mathrm{KOH}$ and the temperature was set at $30^{\circ} \mathrm{C}$. The overpressure in the vessel was kept at 0.3 bar and the stirrer speed was $600 \mathrm{rpm}$, ensuring that the DO was always above $80 \%$. All experiments were carried out with steady-state cultures, that is, after 5 residence times of glucose-limited growth with constant DOT and off-gas readings.

\subsection{Samples for intracellular metabolites (IC)}

Samples were taken using a specialized rapid-sampling setup (Lange et al. 2001). Unless stated otherwise, approximately $1 \mathrm{~g}( \pm 0.05)$ of broth was withdrawn and injected $(\leq 0.8 \mathrm{~s})$ into a tube containing $5 \mathrm{ml} 60 \%$ aqueous methanol (v/v) solution pre-cooled to $-40^{\circ} \mathrm{C}$, the contents of the tube were quickly mixed by vortexing $(\approx 1 \mathrm{~s})$ and the tube was placed back in the cryostat at $-40^{\circ} \mathrm{C}$ (Lauda, Germany). This way, a set of replicate samples can be taken quickly without noticeably disturbing the steady-state of the culture. During all subsequent steps the temperature of the tubes was maintained as close to $-40^{\circ} \mathrm{C}$ as possible. The exact sample weights were determined by weighing each tube before and after sampling. The tubes were centrifuged using a rotor precooled to $-40^{\circ} \mathrm{C}$ at $4,000 \mathrm{~g}$ for $5 \mathrm{~min}$ in a centrifuge cooled to $-20^{\circ} \mathrm{C}$. Unless stated otherwise, the biomass pellets were washed in $5 \mathrm{ml} 60 \%$ methanol (v/v) solution pre-cooled to $-40^{\circ} \mathrm{C}$ and centrifuged again as before. After decanting, $\mathrm{U}-{ }^{13} \mathrm{C}$-labeled cell extract was added to the cell pellets as internal standard (Mashego et al. 2004; Wu et al. 2005).

\subsection{Metabolite extraction}

Extraction of intracellular metabolites was performed using the boiling ethanol method, adapted from (Gonzalez et al. 1997) as described in (Lange et al. 2001). Briefly, each tube was taken from the cryostat at $-40^{\circ} \mathrm{C}$ and $5 \mathrm{ml} 75 \%$ $(\mathrm{v} / \mathrm{v})$ boiling ethanol was added. Each tube was immediately vortexed and placed in a water bath at $95^{\circ} \mathrm{C}$. After 3 min each tube was placed back in the cryostat.

\subsection{Sample concentration}

All ethanol extracts were evaporated under vacuum for $110 \mathrm{~min}$, as described in (Mashego et al. 2004). Dried residues were resuspended in $500 \mu \mathrm{l}$ demineralized water and centrifuged at $15,000 \mathrm{~g}$ for $5 \mathrm{~min}$ at $4^{\circ} \mathrm{C}$. The supernatants were stored at $-80^{\circ} \mathrm{C}$ until analysis.

\subsection{Samples for quenching solution (QS) and washing solution (WS) metabolites}

Instead of discarding the methanol supernatants after quenching or washing, they were collected in pre-cooled tubes at $-40^{\circ} \mathrm{C}$. Each methanol solution was thoroughly vortexed and 300-500 $\mu \mathrm{l}$ was transferred to an empty pre-cooled tube. The exact sample weights were determined by weighing all tubes before and after transfer and keeping record of the corresponding intracellular samples. $\mathrm{U}_{-}{ }^{13} \mathrm{C}$-labeled cell extract was added as internal standard. Boiling ethanol extraction was performed as above to minimize the chance of sample matrix effects and ensure inactivation of any enzyme activity.

\subsection{Samples for whole-broth $(\mathrm{T})$ metabolites}

Sampling was done as for intracellular metabolites but the quenched cell suspension was not centrifuged. Instead, it was thoroughly vortexed and $300-500 \mu \mathrm{l}$ was transferred to an empty tube pre-cooled to $-40^{\circ} \mathrm{C}$. The exact sample weights were determined by weighing all tubes before and after sampling and transfer. $\mathrm{U}^{13} \mathrm{C}$-labeled cell extract was added as internal standard. Boiling ethanol extraction was performed as above to minimize the chance of sample matrix effects and ensure complete cell disruption and inactivation of enzyme activity.

\subsection{Samples for extracellular (EX) metabolites}

Broth was quickly sampled by over-pressure into a syringe containing an amount of cooled steel beads designed to bring the temperature down to $0^{\circ} \mathrm{C}$ (Mashego et al. 2003). The broth was then quickly filtered through a $0.45 \mu \mathrm{m}$ cartridge filter directly into a tube containing $5 \mathrm{ml} 60 \%$ methanol (v/v) at $-40^{\circ} \mathrm{C}$. The resulting methanol filtrate solution was thoroughly vortexed and $300-500 \mu \mathrm{l}$ was transferred to an empty tube pre-cooled to $-40^{\circ} \mathrm{C}$. The exact sample weights were determined by weighing all tubes before and after sampling and transfer. $\mathrm{U}_{-13}^{13} \mathrm{C}$-labeled cell extract was added as internal standard. Boiling ethanol extraction was performed as above to minimize the chance of sample matrix effects and ensure inactivation of any enzyme activity.

\subsection{Metabolite analysis}

The concentrations of the metabolic intermediates G6P, F6P, FBP, PEP, pyruvate, T6P, 6PG, G1P, M6P, citrate, oxoglutarate, succinate, fumarate, and malate, as well as 
the combined pool 2PG $+3 \mathrm{PG}$, were determined by ESILC-MS/MS (Van Dam et al. 2002). The concentrations of several aminoacids were determined by GC-MS using the EZ:Faast kit for free aminoacid analysis from Phenomenex (Torrance, CA, USA). Quantification of the metabolites was based on the use of $\mathrm{U}^{13} \mathrm{C}$-labeled cell extract as internal standard (Mashego et al. 2004; Wu et al. 2005).

\section{Results and discussion}

\subsection{Effect of prolonged exposure}

Our initial experiments aimed at reproducing the results of De Koning and Van Dam, who ruled out the occurrence of leakage because measured metabolite levels did not decrease significantly after $30 \mathrm{~min}$ of extra exposure to the quenching methanol solution (Dekoning and Vandam 1992). We tested this by placing samples back in the cryostat after re-suspension in the washing methanol solution and leaving them at $-40^{\circ} \mathrm{C}$ for periods of +0 , $+30,+60$ or +90 min prior to the second centrifugation step. Unlike the majority of the experiments reported here, this was done with samples from cultures at $D=0.05 \mathrm{~h}^{-1}$ and a biomass concentration of $14.5 \mathrm{~g}_{\mathrm{DW}} / 1$ (Kresnowati et al. 2006). If leakage did not occur, we would expect to find the same intracellular levels regardless of the time of exposure. The results are shown in Fig. 1. G6P $(\mathrm{MW}=260)$ and Fumarate $(\mathrm{MW}=116)$ are given as representative examples of the results found for phosphorylated intermediates (larger, more polar) and organic acids (smaller, less polar), respectively. The results showed that the longer the cells remained in contact with the washing solution, the lower the measured intracellular

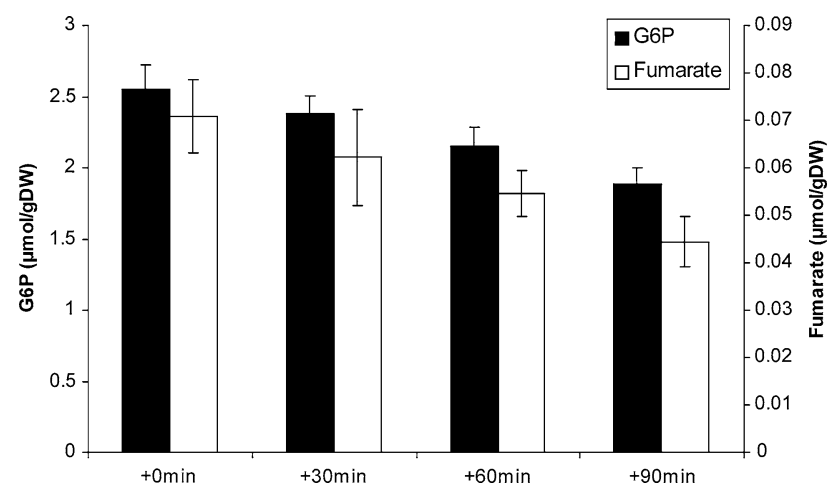

Fig. 1 Effect of prolonged exposure to methanol washing solution $(60 \% \mathrm{v} / \mathrm{v})$ on the measured intracellular levels, exemplified for G6P and fumarate. Prolonged exposure was achieved by leaving the samples at $-40^{\circ} \mathrm{C}$ before the second centrifugation step. Samples were from a culture at $D=0.05 \mathrm{~h}^{-1}$ and $14.5 \mathrm{~g}_{\mathrm{Dw}} / 1$ (Kresnowati et al. 2006). Data are averages \pm standard deviation of four replicate samples, each analyzed in duplicate levels, suggesting time-dependent release of the intracellular metabolites. Interestingly, the rate of decrease was somewhat higher for smaller compounds, which might be released more easily, than for larger compounds. All samples were replicates, taken, treated and analyzed as one sample-set. Sample matrix effects in the analysis can be discarded since $\mathrm{U}_{-}{ }^{13} \mathrm{C}$-labelled extract was used as internal standard. Then, differences can only originate from the period of exposure to the methanol washing solution. These results are not necessarily in contradiction with the work of De Koning and Van Dam, since the measured losses represented not more than $30 \%$ per hour (so $<15 \%$ in $30 \mathrm{~min}$ ), which were probably not observable with the enzymebased analytical techniques available at the time. Nevertheless, they do indicate that leakage is occurring and that the contact time with the methanol solution should be kept to a minimum. That is broadly in agreement with the findings of Villas-Boas et al. (2005). What cannot be concluded from these results is whether significant losses occur even without prolonged exposure, since it cannot be assumed that the rate of loss is the same throughout the entire procedure.

\subsection{Effect of buffers and ionic strength}

Although the original method proposed by De Koning and Van Dam described the use of non-buffered methanol solutions for quenching and washing, much of the later literature describes the use of methanol solutions with some sort of additive, usually a buffer (Ruijter and Visser 1996; Gonzalez et al. 1997). Presumably, this would prevent or minimize cell damage caused by changes in $\mathrm{pH}$ or osmotic shock. However, to our knowledge no quantitative data has ever been presented to back up this claim. We therefore tested the effect of adding buffers (HEPES at $\mathrm{pH}$ 5 or Tricine at $\mathrm{pH}$ 6) or salts $\left(\mathrm{NH}_{4} \mathrm{HCO}_{3}, \mathrm{pH}\right.$ 8), at two different concentrations (10 or $100 \mathrm{mM}$ ), by quenching and washing replicate samples in each type of solution and comparing the metabolite levels measured in the resulting intracellular extracts. If changes in $\mathrm{pH}$ or low ionic strength caused cell damage and losses of metabolites from the cells, we would expect to see higher levels of intracellular metabolites in samples treated with buffers or salts. The results are shown in Fig. 2. Again, G6P and Fumarate are given as representative examples of the results found for phosphorylated intermediates and organic acids, respectively. The results show that there is no significant benefit in buffering or increasing the ionic strength of the methanol solution. On the contrary, for most metabolites this resulted in slightly lower intracellular amounts. In addition, this effect was more pronounced for smaller compounds, which might be released more easily, than for larger compounds. All samples were replicates and $\mathrm{U}_{-}{ }^{13} \mathrm{C}$-labelled extract was 


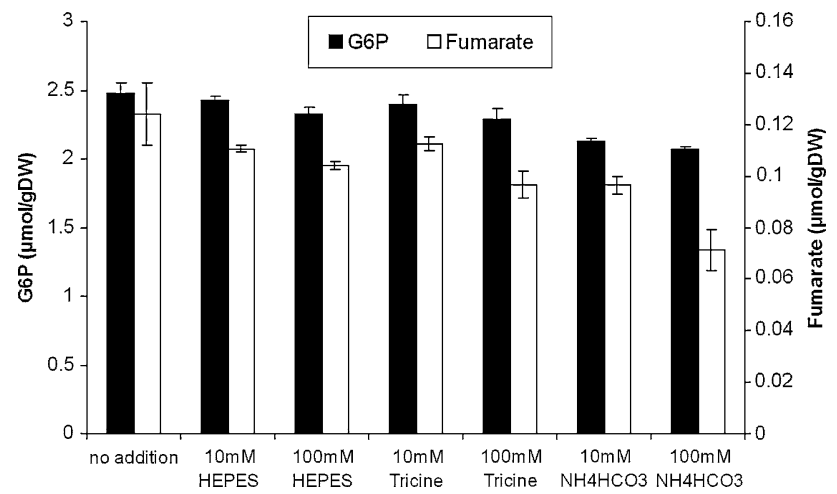

Fig. 2 Effect of additions (HEPES at pH 5, Tricine at $\mathrm{pH} 6$ or $\mathrm{NH}_{4} \mathrm{HCO}_{3}$ at $\mathrm{pH} 8$, to 10 or $100 \mathrm{mM}$ ) to the methanol quenching/ washing solutions $(60 \% \mathrm{v} / \mathrm{v})$ on the measured intracellular levels, exemplified for G6P and fumarate. Samples were from a culture at $D=0.1 \mathrm{~h}^{-1}$ and $3.7 \mathrm{~g}_{\mathrm{DW}} / \mathrm{l}$. Data are averages \pm standard deviation of two replicate samples, each analyzed in duplicate

used as internal standard, so the differences must originate from the quenching and washing steps. These results suggest that losses can occur during quenching/washing, since changing the properties of the methanol solutions can affect the levels found in the cell pellets. Furthermore, they showed that if losses are occurring, they cannot be prevented by adding buffers or salts to the methanol solutions. On the contrary, such additions may even enhance the extent of the losses.

\subsection{Effect of methanol concentration}

Methanol is widely regarded as a toxic compound that can have detrimental effects on cell membrane integrity. A methanol concentration of $60 \%(\mathrm{v} / \mathrm{v})$, as proposed by De Koning and Van Dam, is just enough to keep the cell suspension after sampling $\left(50 \% \mathrm{v} / \mathrm{v}\right.$ final, $\left.\mathrm{FP} \approx-42^{\circ} \mathrm{C}^{1}\right)$ from freezing at $-40^{\circ} \mathrm{C}$. This seems to reflect the perceived need to keep the methanol concentration as low as possible, for fear of causing damage to the cells. We are not aware of subsequent literature where this has been changed. However, to our knowledge no quantitative data has ever been presented to demonstrate the adverse effects of methanol in the context of quenching and intracellular metabolite analysis. We therefore evaluated the effect of the methanol concentration by quenching and washing replicate samples in 50\%, 60\%, 70\% and $80 \%$ methanol and comparing the metabolite levels measured in the resulting intracellular extracts. To avoid freezing of the samples quenched with $50 \%(\mathrm{v} / \mathrm{v})$ solutions $\left(42 \% \mathrm{v} / \mathrm{v}\right.$ final, $\mathrm{FP} \approx-32^{\circ} \mathrm{C}$ (see footnote 1)), in this experiment the cryostat temperature was $-30^{\circ} \mathrm{C}$ instead of $-40^{\circ} \mathrm{C}$. If increasing methanol

\footnotetext{
${ }^{1}$ Interpolated from the data for methanol in (David 2007) using a sixth order polynomial
}

concentrations caused cell membrane damage and this allowed release of intracellular metabolites, we would expect to find higher metabolite levels in samples treated with lower methanol concentrations. The results are shown in Fig. 3. Once again, G6P and Fumarate are given as representative examples of the results found for phosphorylated intermediates and organic acids, respectively. Contrary to our expectations, in samples obtained by quenching and washing with higher methanol concentrations the intracellular metabolites levels were higher. In addition, the differences followed a clear, smooth profile as function of increasing methanol concentration. All samples were replicates and $\mathrm{U}_{-}{ }^{13} \mathrm{C}$-labelled extract was used as internal standard, so the differences must originate from the quenching and washing steps. Like before, the fact that changing the properties of the methanol solutions could affect the obtained intracellular levels of metabolites suggested that losses can occur during quenching/washing. More importantly, the results unexpectedly show that under these conditions higher concentrations of methanol may reduce those losses, rather than increase them.

\subsection{Full mass balance and the importance of methanol concentration and temperature}

Although useful information can be obtained by analyzing only the intracellular samples, as presented above, these provide merely indications. To draw definitive conclusions on the occurrence and the extent of leakage it is necessary to analyze metabolite levels in all the other sample fractions and perform mass balances. Only then can the fate of the metabolites during sample treatment be adequately evaluated. To achieve this, we have analyzed metabolite amounts in six different fractions (see Fig. 4), representing

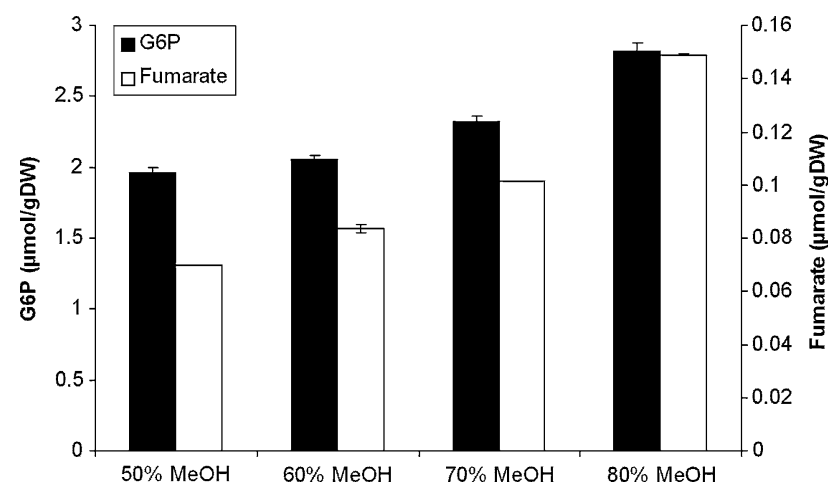

Fig. 3 Effect of the methanol $(\mathrm{MeOH})$ concentration of the quenching/washing solutions on the measured intracellular levels, exemplified for G6P and fumarate. To avoid freezing of the samples quenched with $50 \%$ methanol the cryostat temperature was $-30^{\circ} \mathrm{C}$ instead of $-40^{\circ} \mathrm{C}$. Samples were from a culture at $D=0.1 \mathrm{~h}^{-1}$ and $3.7 \mathrm{~g}_{\mathrm{DW}} / 1$. Data are averages \pm standard deviation of two replicate samples, each analyzed in duplicate 
Fig. 4 Sample fractions where metabolite concentrations were measured to investigate the fate of the metabolites during sample treatment (see Methods for details). The temperature and methanol concentration of the quenching/washing solutions was varied according to Table 1

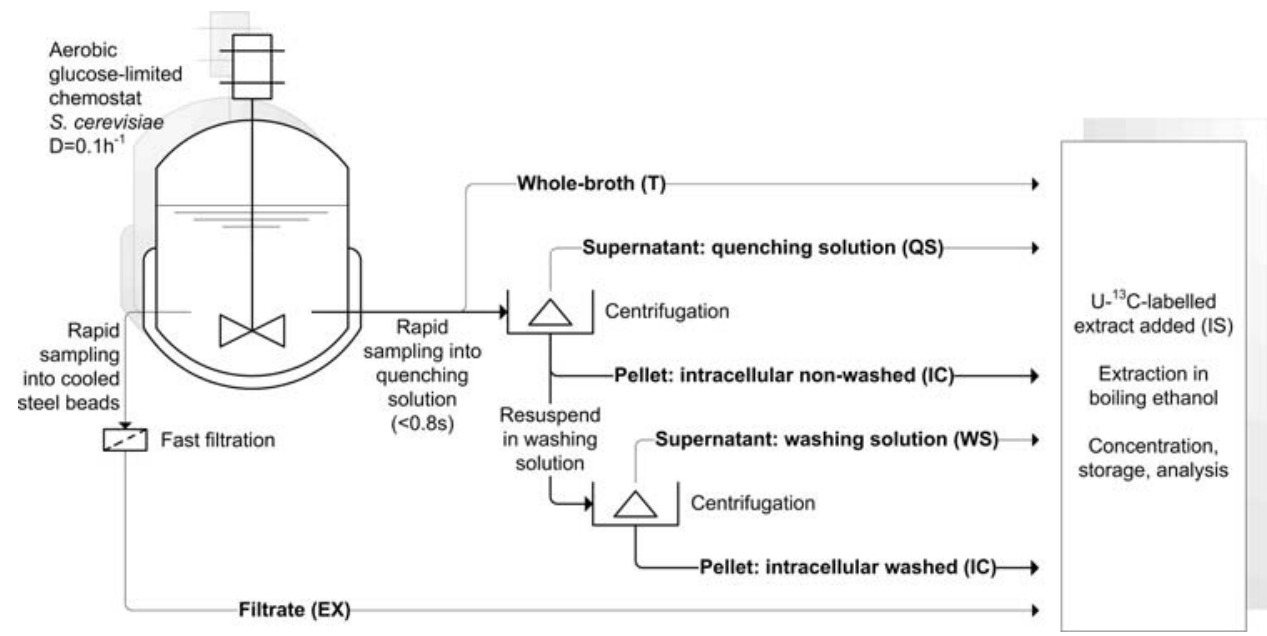

washed and non-washed intracellular samples (IC), the respective quenching and washing methanol solutions (QS and WS), the medium filtrate (EX) and the whole-broth (T). The actual metabolite levels in the cells were estimated by the so-called "differential method" by subtracting the amount in the extracellular medium from the whole-broth total (T - EX). Furthermore, we analyzed all fractions for eight variations of the cold methanol quenching protocol, as described in Table 1, to investigate the effects of methanol concentration and temperature. These samples were analyzed not only for phosphorylated intermediates and organic acids but also for aminoacids, providing an even wider range of compounds. All metabolite levels were expressed in $\mu \mathrm{mol} / \mathrm{g}_{\mathrm{DW}}$, to establish the mass balances. The mass balance results are shown in Fig. 5. G6P and Fumarate are given as representative examples of the results found for phosphorylated intermediates and organic acids and Glutamate $(\mathrm{MW}=147)$ as a representative of midmolecular weight aminoacids. Average mass balance closure over all measured metabolites (calculated as $(\mathrm{IC}+\mathrm{QS}) / \mathrm{T})$ was $105 \%( \pm 36 \%)$, which is quite satisfactory considering the analytical challenge involved. The cell levels calculated by the differential method $(\mathrm{T}-\mathrm{EX})$ can be used as a benchmark, with which the levels measured in the methanol-quenched intracellular samples (IC) can be compared. Detailed data on these values, for each measured metabolite, is provided in Table 2. The $\mathrm{IC} /(\mathrm{T}-\mathrm{EX})$ ratio, which is a measure of how close each protocol variation comes to delivering to "true" intracellular levels, is represented in Fig. 6.

The results show that leakage of intracellular metabolites does occur in the standard procedure of quenching in $60 \%$ methanol at $-40^{\circ} \mathrm{C}$ (Fig. 5), regardless of whether a washing step is carried out (C) or not (D). The measured intracellular levels are lower than the calculated cell levels $(\mathrm{T}-\mathrm{EX})$ and the differences can be found back in the methanol solutions. In the samples that were processed at $-20^{\circ} \mathrm{C}$ instead of $-40^{\circ} \mathrm{C}$, leakage was much more severe (A and $\mathrm{B}$ ). Increasing the final concentration of methanol from $50 \%$ to around $90 \%$ was found to minimize leakage (D-G). At final methanol concentrations above $80 \%$ (F and G) most measured intracellular levels are within $\pm 20 \%$ of the estimated cell levels (Fig. 6). Further decreasing the temperature to $-78^{\circ} \mathrm{C}$ did not result in any significant further improvement $(\mathrm{H})$. In addition, leakage was most critical for smaller metabolites, which may permeate

Table 1 Variations in sample treatment protocol tested to investigate the effects of temperature and methanol concentration on metabolite leakage (see Fig. 4)

\begin{tabular}{llllll}
\hline $\begin{array}{l}\text { Protocol } \\
\text { variation }\end{array}$ & $\begin{array}{l}\text { Temperature } \\
\left({ }^{\circ} \mathrm{C}\right)\end{array}$ & $\begin{array}{l}\text { Concentration of methanol } \\
\text { solutions }(\mathrm{v} / \mathrm{v})(\%)\end{array}$ & $\begin{array}{l}\text { Sample/ } \\
\text { quenching } \\
\text { solution ratio }\end{array}$ & $\begin{array}{l}\text { Concentration of methanol } \\
\text { after sampling }(\mathrm{v} / \mathrm{v})(\%)\end{array}$ \\
\hline A & -20 & 40 & $1: 5$ & 33 & $\begin{array}{l}\text { Washing step } \\
\text { B }\end{array}$ \\
C & -20 & 60 & $1: 5$ & 50 & No \\
D & -40 & 60 & $1: 5$ & 50 & Yes \\
E & -40 & 60 & $1: 5$ & 50 & No \\
F & -40 & 80 & $1: 5$ & 67 & No \\
G & -40 & 100 & $1: 5$ & 83 & No \\
H & -40 & 100 & $1: 10$ & 91 & No \\
\hline
\end{tabular}




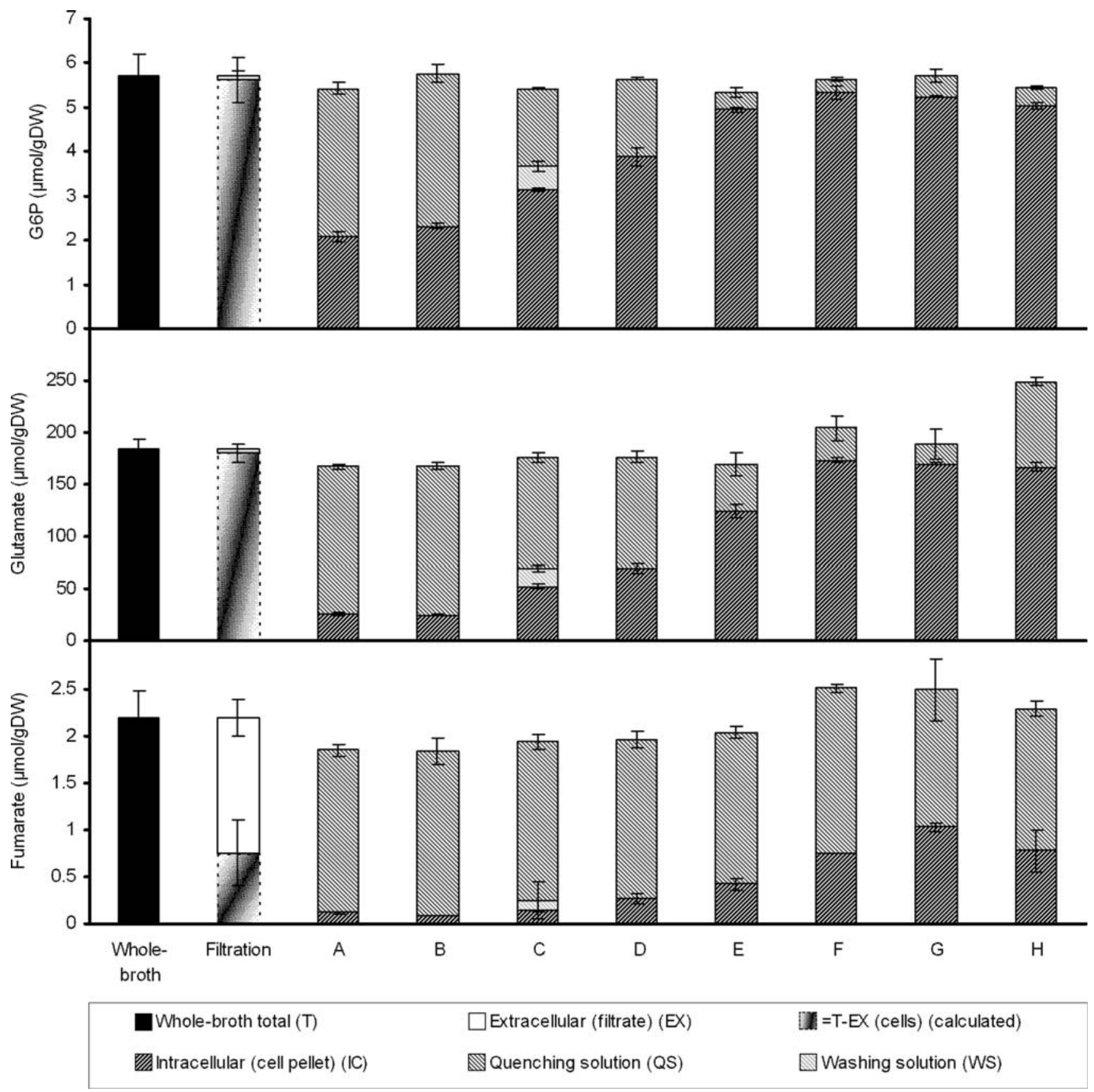

Fig. 5 Full mass balance of the metabolite levels measured in the different sample fractions (see Fig. 4) under eight different variations in sample treatment protocol (see Table 1), exemplified for G6P, glutamate and fumarate. The metabolite levels inside the cells were

through the cell membrane more easily, while bulkier, more polar metabolites seemed to leak less. These results show that the standard protocol of quenching in $60 \%$ methanol at $-40^{\circ} \mathrm{C}$ leads to considerable underestimation of the intracellular levels of most metabolites. The results also highlight the importance of the temperature and the methanol concentration, two factors that were not fully explored before, in obtaining accurate data. Special care estimated from the difference of the concentrations in whole-broth and extracellular medium (T - EX). Samples were from cultures at $D=0.1 \mathrm{~h}^{-1}$ and $3.7 \mathrm{~g}_{\mathrm{Dw}} / \mathrm{l}$. Data are averages \pm standard deviation of at least two replicate samples, each analyzed at least in duplicate

should be taken to ensure that the temperature is not significantly above $-40^{\circ} \mathrm{C}$ throughout the sample treatment, since even at $-20^{\circ} \mathrm{C}$ leakage may become much more severe. Furthermore, the results confirm that at least in the context of quenching, methanol seems to act as a cryopreservant rather than as an extractant and that increasing its final concentration to above $80 \%$ can effectively prevent losses of metabolites from the cells. These conclusions 


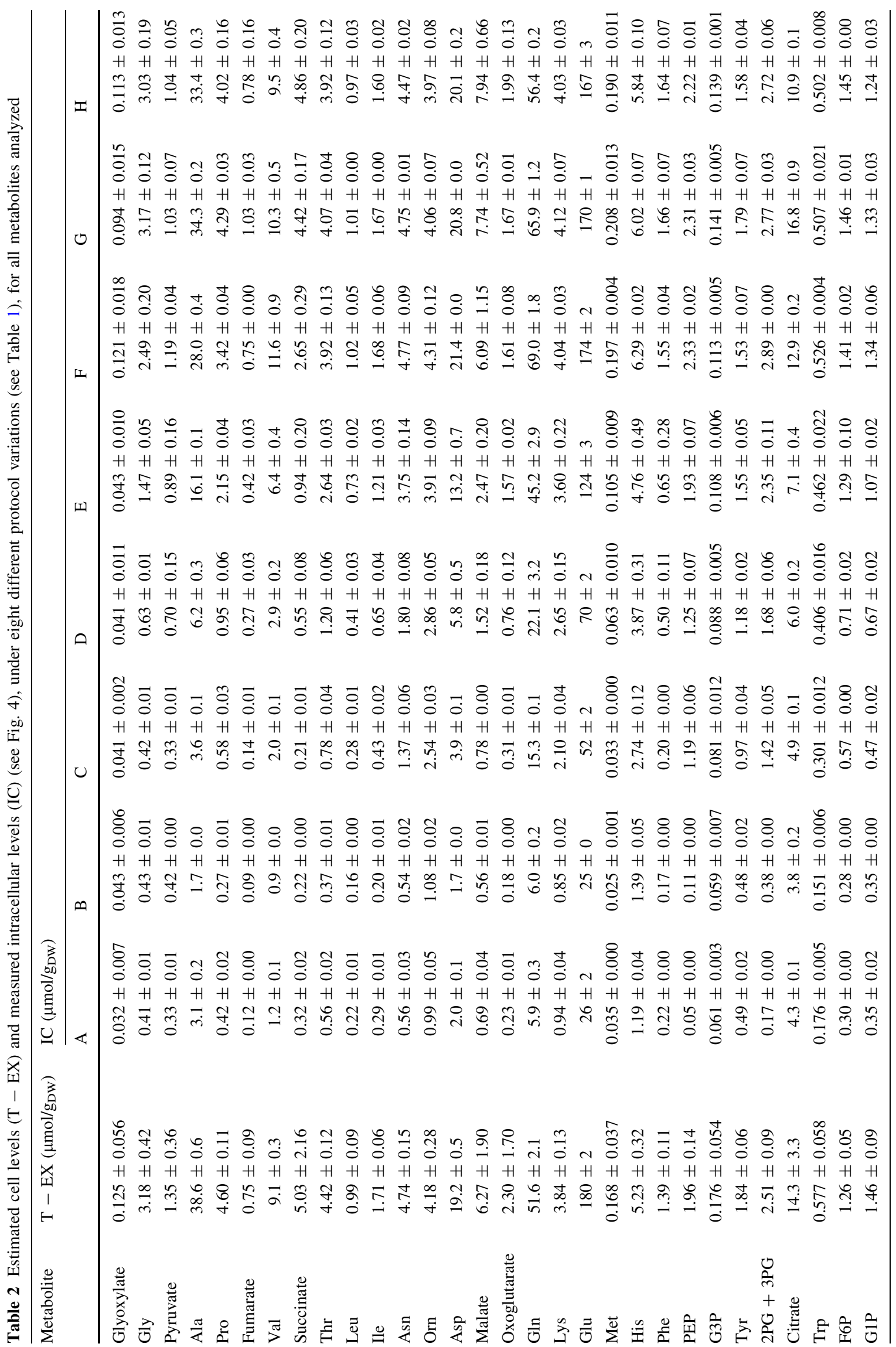



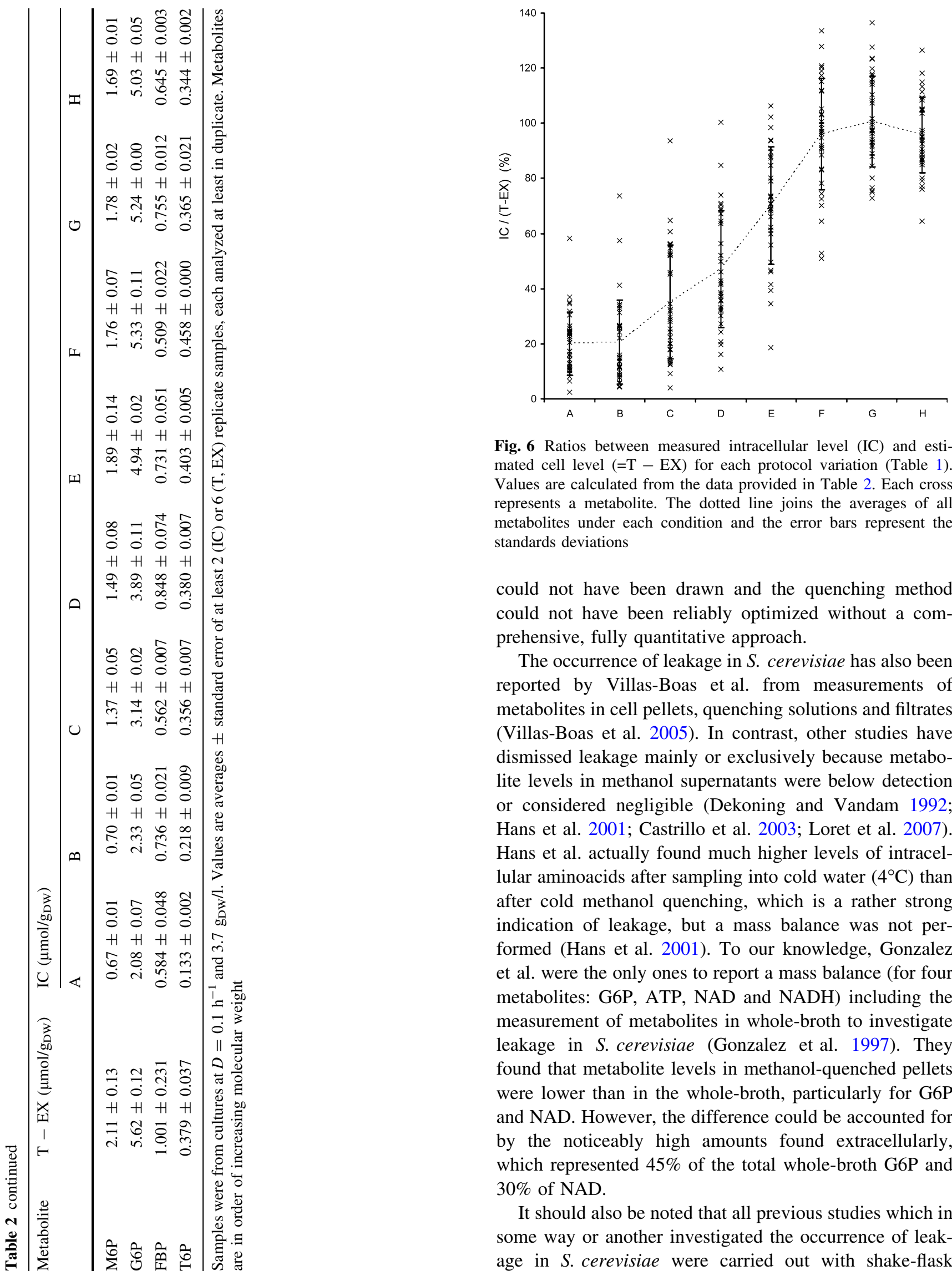

Fig. 6 Ratios between measured intracellular level (IC) and estimated cell level $(=\mathrm{T}-\mathrm{EX})$ for each protocol variation (Table 1). Values are calculated from the data provided in Table 2. Each cross represents a metabolite. The dotted line joins the averages of all metabolites under each condition and the error bars represent the standards deviations

could not have been drawn and the quenching method could not have been reliably optimized without a comprehensive, fully quantitative approach.

The occurrence of leakage in S. cerevisiae has also been reported by Villas-Boas et al. from measurements of metabolites in cell pellets, quenching solutions and filtrates (Villas-Boas et al. 2005). In contrast, other studies have dismissed leakage mainly or exclusively because metabolite levels in methanol supernatants were below detection or considered negligible (Dekoning and Vandam 1992; Hans et al. 2001; Castrillo et al. 2003; Loret et al. 2007). Hans et al. actually found much higher levels of intracellular aminoacids after sampling into cold water $\left(4^{\circ} \mathrm{C}\right)$ than after cold methanol quenching, which is a rather strong indication of leakage, but a mass balance was not performed (Hans et al. 2001). To our knowledge, Gonzalez et al. were the only ones to report a mass balance (for four metabolites: G6P, ATP, NAD and NADH) including the measurement of metabolites in whole-broth to investigate leakage in S. cerevisiae (Gonzalez et al. 1997). They found that metabolite levels in methanol-quenched pellets were lower than in the whole-broth, particularly for G6P and NAD. However, the difference could be accounted for by the noticeably high amounts found extracellularly, which represented $45 \%$ of the total whole-broth G6P and $30 \%$ of NAD.

It should also be noted that all previous studies which in some way or another investigated the occurrence of leakage in S. cerevisiae were carried out with shake-flask 
cultures. Instead, the experiments described in this study were performed in aerobic glucose-limited chemostat cultures. The obvious advantage of chemostat cultures is that they are more reproducible (Hoskisson and Hobbs 2005). In addition, while in batch cultures the time of sampling is critical because metabolite levels are highly dynamic (Hans et al. 2001; Weibel et al. 1974), in steady-state chemostat cultures metabolite levels change much more slowly or not at all (Wu et al. 2006; Mashego et al. 2005). Furthermore, a larger fermenter volume and the use of rapid-sampling equipment mean that a large number of replicate samples can be obtained within a very short period of time and processed together, further improving data consistency and reproducibility. The differences in growth conditions and sampling, as well as differences between S. cerevisiae strains and medium composition are likely to affect the biomass composition and sample properties, which in turn could affect the extent of metabolite leakage. This might explain, at least partly, some of the differences in results.

Our results show that the extent of metabolite leakage depends on the temperature during treatment and the properties of the quenching/washing solutions, namely the methanol concentration and the ionic strength. Throughout all experiments, including tryouts not shown here, the same trends were consistent and reproducible. To our knowledge, none of these factors had been quantitatively investigated before. One possible explanation for the effect of the methanol concentration might be that it decreases the freezing point (FP). The larger the difference between the processing temperature and the freezing point, the lower the chance that part of the sample freezes, causing cell lysis. However, we found that losses by quenching with $40 \%$ methanol $\left(33 \% \mathrm{v} / \mathrm{v}\right.$ final, $\mathrm{FP} \approx-23^{\circ} \mathrm{C}$ (see footnote 1)) and $60 \%$ methanol $\left(50 \% \mathrm{v} / \mathrm{v}\right.$ final, $\mathrm{FP} \approx-42^{\circ} \mathrm{C}$ (see footnote 1)) at $-20^{\circ} \mathrm{C}$ were higher than with $60 \%$ methanol $\left(50 \% \mathrm{v} / \mathrm{v}\right.$ final, $\mathrm{FP} \approx-42^{\circ} \mathrm{C}$ (see footnote 1$)$ ) and $80 \%$ methanol $\left(50 \% \mathrm{v} / \mathrm{v}\right.$ final, $\mathrm{FP} \approx-72^{\circ} \mathrm{C}$ (see footnote 1$)$ ) at $-40^{\circ} \mathrm{C}$, respectively (Fig. $5 \mathrm{~A}$ vs. D and B vs. E). Therefore, metabolite leakage depended both on temperature (Fig. 5B vs. D) and methanol concentration (Fig. 5D vs. E), but not on the difference between temperature and freezing point. In addition, if leakage occurred by cell lysis we would expect to find metabolites levels in the quenching/washing solutions in proportion to their levels inside the cells, which was not the case. What changes in methanol concentration, temperature and ionic strength have in common is that they can affect the solubility and diffusivity of metabolites. Methanol is a fairly polar solvent but it is by far not as good a solvent as water for hydrophilic compounds, so the higher its concentration the lower the solubility of most intracellular metabolites. On the other hand, increasing the ionic strength of a concentrated methanol solution may improve the solubility of polar compounds by allowing more ion-ion interactions. In addition, the solubility and diffusion coefficients usually increase with the temperature. In view of these considerations, we would expect higher methanol concentrations and lower temperatures and lower ionic strengths to reduce the solubility and diffusion rate of polar compounds, which is in agreement with the observed effects in terms of metabolite leakage from the cells. This is also in agreement with the fact that smaller metabolites were found to leak more than larger ones, as illustrated in Fig. 7, since smaller metabolites are expected to have higher diffusivities.

Although increasing the methanol concentration could effectively prevent leakage of intracellular metabolites, this can also have one important disadvantage. We found that samples quenched and washed in solutions with higher methanol content contained much higher levels of phosphate and sulfate. Both are abundant in the medium used for the cultures ( $22 \mathrm{mM}$ phosphate and $40 \mathrm{mM}$ sulfate) and apparently were carried over in the samples, indicating that the washing efficiency was significantly decreased. While leakage can lead to underestimation of intracellular levels, insufficient washing can lead to overestimation. Extracellular components not adequately eliminated can also potentially interfere with certain analytical techniques (e.g. ion-suppression in MS-based analysis). Our results indicate that the intracellular levels were not overestimated even in samples quenched in pure methanol, since the measured IC levels were not consistently higher than the calculated cell levels (T - EX) (Fig. 6F-H). However, in other culture conditions

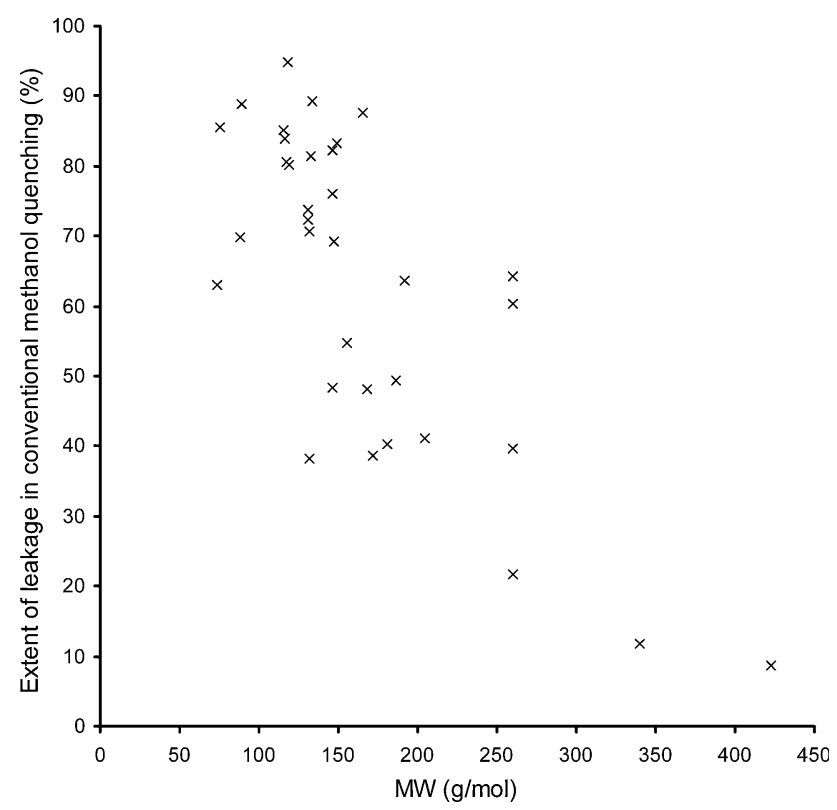

Fig. 7 Relation between the molecular weight (proxy for molecular size) and the extent of leakage under the conventional cold methanol quenching $\left(60 \% \mathrm{v} / \mathrm{v},-40^{\circ} \mathrm{C}\right)$. The extent of leakage was calculated from the data in Table 3 ( 1 minus the inverse of the loss factor) 
where extracellular concentrations are higher (e.g. batch, anaerobic conditions) this may need to be confirmed.

3.5 The importance of quenching metabolism, removing the extracellular medium and avoiding leakage

Although the need to stop metabolic activity upon sampling for intracellular metabolite analysis has been well documented for decades it is still possible to find studies being published which report intracellular metabolite measurements from samples obtained without any apparent concern for quenching. The turnover time, which is the ratio between the metabolite concentration and the flux through that pool, is a good criteria to evaluate the need for quenching. Since our results show that intracellular metabolite levels have been significantly underestimated due to leakage, we have re-calculated the turnover times based on our best estimate of the intracellular levels. These were obtained as the averages of the levels found with quenching in pure methanol (columns F, G and $\mathrm{H}$ of Table 2), where leakage was prevented (Fig. 6). As shown in Table 3, most intermediates in central metabolism have short turnover times, particularly those that are highly connected (Pyruvate) or that have relatively low pools (FBP, fumarate). In this regard it is worth mentioning that cofactors such as ATP, ADP, NAD and NADH, which are involved in large numbers of reactions, are likely to have even shorter turnover times. On the other hand, most aminoacids have much longer turnover times, owing to proportionally small fluxes and large pools. In view of these results we can conclude that sub-second quenching of metabolic activity is necessary for reliable analysis of intracellular levels of intermediates in central metabolism, while slower sampling methods such as filtration (Bolten et al. 2007) may also acceptable for analysis of aminoacid levels.

As mentioned before, the main reason to use cold methanol quenching is that it allows removal of metabolites present in the extracellular medium. This is necessary if a significant fraction of the metabolites is present outside the cells. It may also be desirable in cases where medium components can interfere with analytical techniques (e.g. high salt concentrations can cause peak-shifting in ion-exchange LC and ion-supression in MS). If separation was not necessary, whole-broth extraction might be a simpler and faster alternative. Since we analyzed metabolite levels in all sample fractions, we can re-check the need to separate the extracellular medium by calculating the extracellular fraction for each metabolite $(=\mathrm{EX} / \mathrm{T})$. It should be kept in mind that a large extracellular fraction does not necessarily mean that the extracellular concentration is high. The reason for this is that the extracellular volume is typically 100-1,000fold higher than the intracellular volume so even very low extracellular concentrations can represent a large fraction of the total whole-broth amount (see Fig. 5). As shown in Table 3, several metabolites have rather high extracellular fractions, particularly organic acids and aminoacids with non-polar side chains. Accurate analysis of the intracellular levels of these metabolites therefore requires adequate separation of the extracellular medium, while methods involving whole-broth extraction may be acceptable for analysis of compounds such as the phosphorylated intermediates, which are mostly present inside the cells. Note that these results were obtained in carbon-limited cultures and that extracellular fractions are most likely higher in carbonexcess conditions (Bolten et al. 2007).

One possibility to differentiate between intracellular and extracellular metabolites is the "differential method", which we used above as benchmark for the improvement of the cold methanol quenching protocol. The major disadvantage of the differential method is the propagation of error associated with the subtraction, especially because metabolite levels in the filtrate are very dilute and difficult to assay reliably. This problem is further amplified if the extracellular fraction is large (two large numbers being subtracted to obtain a small number). The only way to try to minimize the uncertainty is through "brute force", by increasing the number of replicate samples (note that the uncertainties in Table 2 are standard errors, not standard deviations), but in practice this is sometimes not possible (e.g. investigation of short-term dynamics) and it is not guaranteed to succeed. The differential method is also not an alternative in the cases where medium components interfere with the analytical procedures.

It follows that the most desirable is to have a sampling method that ensures sub-second quenching of metabolism while allowing separation of the extracellular medium, which explains the popularity of the cold methanol method. Methanol is the most obvious choice of solvent because it is fully miscible with water (in contrast with more apolar solvents) but has a very low freezing-point (much lower than ethanol, propanol or glycerol, for example). In addition, methanol is thought to be less harmful to the cells than other organic compounds and methanol-water solutions are not very viscous, which allows easy centrifugation and cell separation. However, cold methanol quenching can only be used if leakage of intracellular metabolites does not occur. Much of the work reported here aimed at quantifying the extent of leakage and trying to prevent it. As shown in Table 3, the extent of leakage in the conventional cold methanol quenching method $\left(60 \%,-40^{\circ} \mathrm{C}\right)$ is such that most metabolites investigated would be underestimated by more than twofold. With the exception of only the largest, most polar metabolites (FBP, T6P and probably others such as nucleotides), accurate analysis of the intracellular metabolite levels in $S$. cerevisiae is only possible using the improved cold methanol quenching method described here. 
Table 3 Intracellular levels, turnover times, extracellular fractions and loss factors due to leakage during conventional cold methanol quenching

\begin{tabular}{|c|c|c|c|c|c|}
\hline Metabolite & $\begin{array}{l}\text { Intracellular } \\
\text { level }\left(\mu \mathrm{mol} / \mathrm{g}_{\mathrm{DW}}\right)\end{array}$ & $\begin{array}{l}\text { Estimated flux } \\
\left(\mu \mathrm{mol} / \mathrm{g}_{\mathrm{DW}} / \mathrm{s}\right)\end{array}$ & $\begin{array}{l}\text { Estimated } \\
\text { turnover time (s) }\end{array}$ & $\begin{array}{l}\text { Extracellular } \\
\text { fraction }(\%)\end{array}$ & $\begin{array}{l}\text { Loss factor due to leakage } \\
\text { in conventional cold } \\
\text { methanol quenching }\end{array}$ \\
\hline Glyoxylate & 0.11 & - & - & 70 & 2.7 \\
\hline Gly & 2.9 & 0.012 & 247 & 33 & 6.9 \\
\hline Pyruvate & 1.1 & 0.656 & 2 & 57 & 3.3 \\
\hline Ala & 32 & 0.010 & 3268 & 5 & 8.9 \\
\hline Pro & 3.9 & 0.004 & 925 & 8 & 6.7 \\
\hline Fumarate & 0.85 & 0.208 & 4 & 66 & 6.2 \\
\hline Val & 10 & 0.021 & 490 & 6 & 5.1 \\
\hline Succinate & 4.0 & 0.197 & 20 & 50 & 19.2 \\
\hline Thr & 4.0 & 0.018 & 220 & 9 & 5.1 \\
\hline Leu & 1.0 & 0.008 & 125 & 28 & 3.6 \\
\hline Ile & 1.6 & 0.012 & 140 & 15 & 3.8 \\
\hline Asn & 4.7 & 0.004 & 1142 & 9 & 3.4 \\
\hline Orn & 4.1 & 0.008 & 502 & 22 & 1.6 \\
\hline Asp & 21 & 0.036 & 577 & 4 & 5.3 \\
\hline Malate & 7.3 & 0.240 & 30 & 61 & 9.3 \\
\hline Oxoglutarate & 1.8 & 0.377 & 5 & 78 & 5.6 \\
\hline Gln & 64 & 0.027 & 2401 & 3 & 4.2 \\
\hline Lys & 4.1 & 0.007 & 619 & 8 & 1.9 \\
\hline Glu & 170 & 0.153 & 1112 & 2 & 3.3 \\
\hline Met & 0.20 & 0.003 & 66 & 42 & 6.0 \\
\hline His & 6.0 & 0.002 & 3141 & 10 & 2.2 \\
\hline Phe & 1.6 & 0.004 & 430 & 17 & 8.1 \\
\hline PEP & 2.3 & 0.404 & 6 & 8 & 1.9 \\
\hline G3P & 0.13 & 0.002 & 57 & 70 & 1.6 \\
\hline Tyr & 1.6 & 0.002 & 832 & 11 & 1.7 \\
\hline $2 \mathrm{PG}+3 \mathrm{PG}$ & 2.8 & 0.422 & 7 & 8 & 2.0 \\
\hline Citrate & 14 & 0.228 & 59 & 50 & 2.8 \\
\hline Trp & 0.51 & 0.001 & 788 & 17 & 1.7 \\
\hline F6P & 1.4 & 0.197 & 7 & 2 & 2.5 \\
\hline G1P & 1.3 & - & - & 20 & 2.8 \\
\hline M6P & 1.7 & - & - & 1 & 1.3 \\
\hline G6P & 5.2 & 0.311 & 17 & 2 & 1.7 \\
\hline FBP & 0.64 & 0.197 & 3 & 6 & 1.1 \\
\hline T6P & 0.39 & - & - & 16 & 1.1 \\
\hline
\end{tabular}

Best estimates of the intracellular levels are the averages of levels obtained by quenching in pure methanol (average of columns $\mathrm{F}, \mathrm{G}$ and $\mathrm{H}$ of Table 2). Net fluxes through metabolite pools were calculated from the estimated steady-state intracellular flux distribution under aerobic glucose-limited conditions according to (Daran-Lapujade et al. 2004). The turnover time is the ratio between the intracellular level and the flux. The extracellular fraction is the ratio between the levels measured in the extracellular (EX) and total broth (T) samples. The loss factor due to leakage is the ratio between the best estimate of the intracellular level (in the second column) and the level found by conventional cold methanol quenching (column $\mathrm{C}$ of Table 2). Metabolites are in order of increasing molecular weight

-, Metabolites not present in the stoichiometric model (Daran-Lapujade et al. 2004) under aerobic glucose-limited conditions

These findings have obvious implications for metabolomics-based research in S. cerevisiae, such as nonstationary ${ }^{13} \mathrm{C}$ flux analysis, in vivo kinetic modeling and thermodynamic network analysis, which all rely on the accuracy of intracellular metabolite determination.

\section{Concluding remarks and recommendations}

For accurate determination of intracellular metabolites in $S$. cerevisiae broth should be harvested into pure methanol at $\leq-40^{\circ} \mathrm{C}$ with a sample/quenching solution ratio of 1:5 
or lower (final methanol concentration $\geq 83 \%$ ). Preferably, this should be done using rapid sampling equipment to avoid changes in metabolite concentrations during harvesting, especially from chemostat cultures and if shortturnover metabolites are to be measured. Centrifugation should be done quickly ( $\leq 5 \mathrm{~min}$ at $\geq 4,000 \mathrm{~g}$ ) and using a pre-cooled rotor, to keep the temperature below or around $-40^{\circ} \mathrm{C}$. The overall time of exposure to methanol should be kept to a minimum. If a washing step is strictly necessary, to minimize the concentration of contaminants from the extracellular medium, this should be done with $\geq 83 \%$ methanol.

Sample treatment techniques should be validated quantitatively for each microorganism and growth condition. Not doing so compromises the conclusions drawn from subsequent research.

Acknowledgement This work was funded by SenterNovem through the IOP Genomics initiative (project IGE3006A).

Open Access This article is distributed under the terms of the Creative Commons Attribution Noncommercial License which permits any noncommercial use, distribution, and reproduction in any medium, provided the original author(s) and source are credited.

\section{References}

Bolten, C. J., Kiefer, P., Letisse, F., Portais, J. C., \& Wittmann, C. (2007). Sampling for metabolome analysis of microorganisms. Analytical Chemistry, 79, 3843-3849. doi:10.1021/ac0623888.

Castrillo, J. I., Hayes, A., Mohammed, S., Gaskell, S. J., \& Oliver, S. G. (2003). An optimized protocol for metabolome analysis in yeast using direct infusion electrospray mass spectrometry. Phytochemistry, 62, 929-937. doi:10.1016/S0031-9422(02)00713-6.

Cole, H. A., Wimpenny, J. W., \& Hughes, D. E. (1967). Atp pool in Escherichia Coli. I. Measurement of pool using a modified luciferase assay. Biochimica et Biophysica Acta 143, 445-453.

Daran-Lapujade, P, Jansen, M. L. A., Daran, J. M., van Gulik, W., de Winde, J. H., \& Pronk, J. T. (2004) Role of transcriptional regulation in controlling fluxes in central carbon metabolism of Saccharomyces cerevisiae-A chemostat culture study. The Journal of Biological Chemistry, 279, 9125-9138.

David, R. L. (2007) Concentrative properties of aqueous solutions: Density, refractive index, freezing point depression, and viscosity. In R. L. David (Ed.), CRc handbook of chemistry and physics, internet version 2007 (87th ed.). Boca Raton, FL: Taylor and Francis.

Dekoning, W., \& Vandam, K. (1992). A method for the determination of changes of glycolytic metabolites in yeast on a subsecond time scale using extraction at neutral $\mathrm{Ph}$. Analytical Biochemistry, 204, 118-123. doi:10.1016/0003-2697(92)90149-2.

Faupel, R. P., Seitz, H. J., Tarnowsk, W., Thiemann, V., \& Weiss, C. (1972). Problem of tissue sampling from experimental-animals with respect to freezing technique, anoxia, stress and narcosisNew method for sampling rat-liver tissue and physiological values of glycolytic intermediates and related compounds. Archives of Biochemistry and Biophysics, 148, 509-522.

Gonzalez, B, Francois, J, \& Renaud, M. (1997). A rapid and reliable method for metabolite extraction in yeast using boiling buffered ethanol. Yeast (Chichester, England), 13, 1347-1355.

Hajjaj, H., Blanc, P. J., Goma, G., \& Francois, J. (1998). Sampling techniques and comparative extraction procedures for quantitative determination of intra- and extracellular metabolites in filamentous fungi. FEMS Microbiology Letters, 164, 195-200. doi:10.1111/j.1574-6968.1998.tb13085.x.

Hans, M. A., Heinzle, E., \& Wittmann, C. (2001). Quantification of intracellular amino acids in batch cultures of Saccharomyces cerevisiae. Applied Microbiology and Biotechnology, 56, 776779. doi: $10.1007 / \mathrm{s} 002530100708$.

Harrison, D. E., \& Maitra, P. K. (1969). Control of respiration and metabolism in growig klebsiella aerogenes-Role of adenine nucleotides. Biochemical Journal, 112, 647-656.

Hoskisson, P. A., \& Hobbs, G. (2005). Continuous culture-Making a comeback? Microbiology-Sgm, 151, 3153-3159.

Kresnowati, M. T. A. P, Van Winden, W. A, Almering, M. J. H, ten Pierick, A, Ras, C, Knijnenburg, T. A., et al. (2006). When transcriptome meets metabolome: Fast cellular responses of yeast to sudden relief of glucose limitation. Molecular Systems Biology, 2, 49.

Lange, H. C., Eman, M., van Zuijlen, G., Visser, D., Van Dam, J. C., Frank, J., et al. (2001). Improved rapid sampling for in vivo kinetics of intracellular metabolites in Saccharomyces cerevisiae. Biotechnology and Bioengineering, 75, 406-415. doi: 10.1002/bit.10048.

Loret, M. O., Pedersen, L., \& Francois, J. (2007). Revised procedures for yeast metabolites extraction: Application to a glucose pulse to carbon-limited yeast cultures, which reveals a transient activation of the purine salvage pathway. Yeast (Chichester, England), 24, 47-60. doi:10.1002/yea.1435.

Maharjan, R. P., \& Ferenci, T. (2003). Global metabolite analysis: The influence of extraction methodology on metabolome profiles of Escherichia coli. Analytical Biochemistry, 313, 145-154. doi: 10.1016/S0003-2697(02)00536-5.

Mashego, M. R., Jansen, M. L. A., Vinke, J. L., Van Gulik, W. M., \& Heijnen, J. J. (2005). Changes in the metabolome of Saccharomyces cerevisiae associated with evolution in aerobic glucoselimited chemostats. FEMS Yeast Research, 5, 419-430. doi: 10.1016/j.femsyr.2004.11.008.

Mashego, M. R., Rumbold, K., De Mey, M., Vandamme, E., Soetaert, W., \& Heijnen, J. J. (2007). Microbial metabolomics: Past, present and future methodologies. Biotechnology Letters, 29, 116. doi:10.1007/s10529-006-9218-0.

Mashego, M. R., Van Gulik, W. M., Vinke, J. L., \& Heijnen, J. J. (2003). Critical evaluation of sampling techniques for residual glucose determination in carbon-limited chemostat culture of Saccharomyces cerevisiae. Biotechnology and Bioengineering, 83, 395-399. doi:10.1002/bit.10683.

Mashego, M. R., Wu, L., Van Dam, J. C., Ras, C., Vinke, J. L., Van Winden, W. A., et al. (2004). MIRACLE: Mass isotopomer ratio analysis of U-C-13-labeled extracts. A new method for accurate quantification of changes in concentrations of intracellular metabolites. Biotechnology and Bioengineering, 85, 620-628. doi:10.1002/bit.10907.

Moritz, B., Striegel, K., de Graaf, A. A., \& Sahm, H. (2000). Kinetic properties of the glucose-6-phosphate and 6-phosphogluconate dehydrogenases from Corynebacterium glutamicum and their application for predicting pentose phosphate pathway flux in vivo. European Journal of Biochemistry, 267, 3442-3452. doi:10.1046/j.1432-1327.2000.01354.x.

Oldiges, M., \& Takors, R. (2005) Applying metabolic profiling techniques for stimulus-response experiments: Chances and pitfalls. Technology Transfer in Biotechnology: From Lab to Industry to Production, 92, 173-196.

Ruijter, G. J. G., \& Visser, J. (1996). Determination of intermediary metabolites in Aspergillus niger. Journal of Microbiological Methods, 25, 295-302. doi:10.1016/0167-7012(95)00104-2.

Saez, M. J., \& Lagunas, R. (1976). Determination of intermediary metabolites in yeast-Critical-examination of effect of sampling 
conditions and recommendations for obtaining true levels. Molecular and Cellular Biochemistry, 13, 73-78. doi:10.1007/ BF01837056.

Schaub, J., Schiesling, C., Reuss, M., \& Dauner, M. (2006). Integrated sampling procedure for metabolome analysis. Biotechnology Progress, 22, 1434-1442. doi:10.1021/bp050381q.

Van Dam, J. C., Eman, M. R., Frank, J., Lange, H. C., van Dedem, G. W. K., \& Heijnen, S. J. (2002). Analysis of glycolytic intermediates in Saccharomyces cerevisiae using anion exchange chromatography and electrospray ionization with tandem mass spectrometric detection. Analytica Chimica Acta, 460, 209-218. doi:10.1016/S0003-2670(02)00240-4.

van Dijken, J. P., Bauer, J., Brambilla, L., Duboc, P., Francois, J. M., Gancedo, C., et al. (2000). An interlaboratory comparison of physiological and genetic properties of four Saccharomyces cerevisiae strains. Enzyme and Microbial Technology, 26, 706714. doi:10.1016/S0141-0229(00)00162-9.

Veech, R. L., Egglesto, L. V., \& Krebs, H. A. (1969). Redox state of free nicotinamide-adenine dinucleotide phosphate in cytoplasm of rat liver. Biochemical Journal, 115, 609-619.

Verduyn, C., Postma, E., Scheffers, W. A., \& Vandijken, J. P. (1992). Effect of benzoic-acid on metabolic fluxes in yeasts-A continuous-culture study on the regulation of respiration and alcoholic fermentation. Yeast (Chichester, England), 8, 501-517.

Villas-Boas, S. G., \& Bruheim, P. (2007). Cold glycerol-saline: The promising quenching solution for accurate intracellular metabolite analysis of microbial cells. Analytical Biochemistry, 370, 87-97.

Villas-Boas, S. G., Hojer-Pedersen, J., Akesson, M., Smedsgaard, J., \& Nielsen, J. (2005). Global metabolite analysis of yeast:
Evaluation of sample preparation methods. Yeast (Chichester, England), 22, 1155-1169. doi:10.1002/yea.1308.

Weibel, K. E., Mor, J. R., \& Fiechter, A. (1974). Rapid sampling of yeast-cells and automated assays of adenylate, citrate, pyruvate and glucose-6-phosphate pools. Analytical Biochemistry, 58, 208-216. doi:10.1016/0003-2697(74)90459-X.

Williams, D. H., Lund, P., \& Krebs, H. A. (1967). Redox state of free nicotinamide-adenine dinucleotide in cytoplasm and mitochondria of rat liver. Biochemical Journal, 103, 514-527.

Wittmann, C, Kromer, J. O., Kiefer, P., Binz, T., \& Heinzle, E. (2004). Impact of the cold shock phenomenon on quantification of intracellular metabolites in bacteria. Analytical Biochemistry, 327, 135-139.

Wollenberger, A., Ristau, O., \& Schoffa, G. (1960). Eine Einfache Technik der Extrem Schnellen Abkuhlung Grosserer Gewebestucke. Pflugers Archiv fur die Gesamte Physiologie des Menschen und der Tiere, 270, 399-412. doi:10.1007/BF00 362995.

Wu, L., Mashego, M. R., Proell, A. M., Vinke, J. L., Ras, C., van Dam, J., et al. (2006). In vivo kinetics of primary metabolism in Saccharomyces cerevisiae studied through prolonged chemostat cultivation. Metabolic Engineering, 8, 160-171. doi: 10.1016/j.ymben.2005.09.005.

Wu, L., Mashego, M. R., Van Dam, J. C., Proell, A. M., Vinke, J. L., Ras, C., et al. (2005). Quantitative analysis of the microbial metabolome by isotope dilution mass spectrometry using uniformly C-13-labeled cell extracts as internal standards. Analytical Biochemistry, 336, 164-171. doi:10.1016/j.ab. 2004.09.001. 